\title{
Ionospheric trends in Southern Hemisphere stations due to the increasing greenhouse gases concentration
}

\author{
José V. Venchiarutti ${ }^{1}$, Blas F. de Haro ${ }^{1, *}$, Ana G. Elías ${ }^{1,2}$ \\ ${ }^{1}$ Universidad Nacional de Tucumán, Departamento de Física, Tucumán, Argentina \\ ${ }^{2}$ Consejo Nacional de Investigaciones Cientificas y Técnicas, CONICET, Argentina
}

\author{
Article history \\ Received December 3, 2014; accepted May 22, 2015. \\ Subject classification: \\ Ionospheric trend, EUV proxies, Forecasts, Dynamics.
}

\begin{abstract}
The lower and middle atmosphere present long-term variations in temperature and other parameters linked to anthropogenic sources, such as the increase in greenhouse gases concentration since the start of the industrial era. Some examples are the well known temperature increase in the troposphere and stratosphere cooling. Upper atmosphere parameters also present long-term variations. While in the case of the middle and lower atmosphere it is debated whether the origin of the trends is primarily anthropogenic or solar, in the upper atmosphere other sources are also able to induce long-term changes, such as long-term variations in geomagnetic activity and secular variations of the Earth's magnetic field. In this paper, trends of the F2 layer critical frequency, foF2, measured at three Southern Hemisphere stations (Brisbane, Canberra and Christchurch) are analyzed to determine the importance of increasing greenhouse gases concentration effect. According to our results for the period covering solar cycles 18 to 21 (period 1944-1986), it is more important than natural forcings. Update estimates including solar cycle 23 are presented although the difficulty due to two of the three stations present big data gaps during cycle 22 and traditional ionospheric filtering is no longer entirely reliable for cycle 23. The aim of this study is to contribute both to an active area of aeronomy as is the study of trends in the upper atmosphere, and to the understanding of climate change.
\end{abstract}

\section{Introduction}

Since the beginning of the 1990s there has been a great interest in the study of long-term trends in the upper atmosphere and several papers have been published since then on this topic (see Lastovicka et al. [2012] for a very good review). Some of these studies associate trends in the ionosphere with cooling in the thermosphere due to increased concentration of greenhouse gases [Roble and Dickinson 1989, Rishbeth 1990]. If the concentration of $\mathrm{CO}_{2}$ is doubling, according to theoretical models a cooling of $30-40 \mathrm{~K}$ in the thermosphere should be expected together with a decrease in air density, a decrease of $15-20 \mathrm{~km}$ of the peak height of the F2 layer, $\mathrm{hmF} 2$, and a decrease in the critical frequency of the F2 layer, foF2, less than $0.5 \mathrm{MHz}$ globally. However the overall pattern of $\mathrm{hmF} 2$ and foF 2 trends estimated with experimental data of several worldwide stations is very complex and would not completely agree with the greenhouse gases hypothesis.

Another possible cause of the ionospheric trends would be the long-term variation of geomagnetic activity [Mikhailov and Marin 2000] and the secular variations of the Earth's intrinsic magnetic field [Foppiano et al. 1999, Elias 2009, Cnossen 2014].

In this paper we estimate foF 2 trends measured at three Southern Hemisphere ionospheric stations, which have records since solar cycle 18 , and analyze them in terms of the cooling expected from the anthropogenic effects. Being able to determine the causes of the observed ionospheric trends, would contribute to the study of variability in the ionosphere for predictive purposes and the problems of global change directly linked to increased concentration of greenhouse gases.

\section{Data analysis}

Monthly median values of foF2 from Damboldt and Suessmann database [2012] (ftp: / /ftp.ips.gov.au/ wdc-data/iondata/medians/Damboldt/) were used at 12 LT. Three Southern Hemisphere stations which are listed in Table 1 were selected which had long enough records and few data gaps.

Canberra is the only that presents an almost uninterrupted record for the period 1941-2009. Christchurch presents a first almost complete period 1941 to1986, and then returns in 1993-2009, while in the case of Brisbane the almost complete data periods are 1943-1986 and 1998-2009. Due to the big data gap in solar cycle 22, trends are estimated first for the period 1944-1986 which covers four complete solar cycles, 18 to 21 . Then 

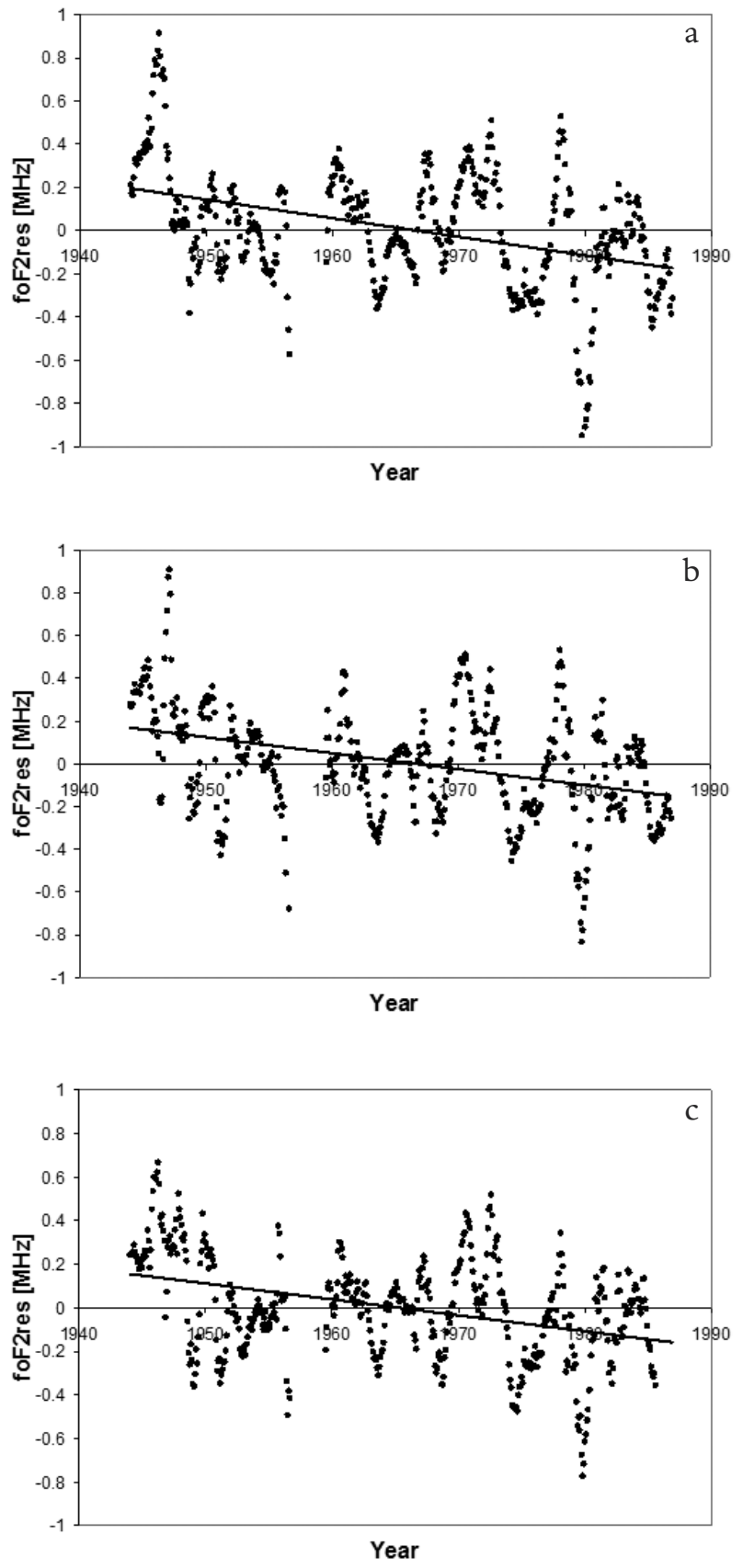

Figure 1. foF2 residual for (a) Brisbaen $\left(27.5^{\circ} \mathrm{S}, 152.9^{\circ} \mathrm{E}\right)$, (b) Canberra $\left(35.3^{\circ} \mathrm{S}, 149.0^{\circ} \mathrm{E}\right)$ and (c) Christchurch $\left(43.6^{\circ} \mathrm{S}, 172.8^{\circ} \mathrm{E}\right)$, after filtering seasonal and solar activity effects using $\mathrm{Rz}$ as solar EUV proxy (black dots) and fitted linear trend (solid line). they are updated to include solar cycle 23 with special care in the filtering process required in ionospheric trend estimation.

\subsection{Trends during period covering solar cycles 18 to 21}

In order to minimize spurious effects introduced by the filtering process here applied [Lastovicka et al. 2012, Elias 2014], trends should be assessed for the same period in all the cases analyzed and include complete solar cycles. In our case this period corresponds to 19441986, that is solar cycles 18 to 21 .

The steps followed to estimate foF 2 trend are:

1. Seasonal variation was filtered out assessing 12month running mean of each data series analyzed.

2. Periods with $\mathrm{Rz}>160$ were eliminated due to saturation effect occurring in the F2 region.

3. Solar cycle 23 was not considered due to lack of a close agreement between $\mathrm{Rz}$ (or F10.7) and solar EUV [Emmert et al. 2010, Chen et al. 2011, Tapping and Valdes 2011]. Its inclusion is analyzed in the following section.

3. Solar activity effect was filtered out estimating the residuals from the regression between the experimental foF2 values and the solar activity index Rz, that is:

$$
\mathrm{foF} 2_{\text {res }}=\mathrm{foF} 2_{\exp }-(\mathrm{a}+\mathrm{b} \mathrm{Rz})
$$

where foF $2_{\text {exp }}$ is the experimental foF 2 value, $\mathrm{Rz}$ is the sunspot number used as a proxy for the solar EUV radiation, and $\mathrm{a}$ and $\mathrm{b}$ are the regression constants assessed with least squares.

4. The linear trend $\alpha$ (in $\mathrm{MHz} /$ year) is calculated from

$$
\text { foF } 2_{\text {res }}=\alpha \mathrm{t}+\beta
$$

Figure 1 shows foF $2_{\text {res }}$ together with the fitted linear trend. Table 1 shows the values of the trends obtained. Trend errors in all the cases are around $8-11 \%$ of trend values, that is $\sim 6-9 \times 10^{-4} \mathrm{Mhz} /$ year. All trend val-

\begin{tabular}{|c|c|c|c|c|c|c|}
\hline \multirow{3}{*}{ Station } & \multirow{3}{*}{$\begin{array}{l}\text { Geographic } \\
\text { latitude }\end{array}$} & \multirow{3}{*}{$\begin{array}{l}\text { Geographic } \\
\text { longitude }\end{array}$} & \multicolumn{4}{|c|}{ foF 2 trend $[\mathrm{MHz} /$ year $]$} \\
\hline & & & \multicolumn{2}{|c|}{ Period cycles 18-21 } & \multicolumn{2}{|c|}{ Period cycles 18-23 } \\
\hline & & & $\mathrm{Rz}$ as proxy & F10.7 as proxy & $\mathrm{Rz}$ as proxy & F10.7 as proxy \\
\hline Brisbaen & $27.5^{\circ} \mathrm{S}$ & $152.9^{\circ} \mathrm{E}$ & -0.0086 & -0.0120 & $+0.0004^{\star}$ & -0.0102 \\
\hline Canberra & $35.3^{\circ} \mathrm{S}$ & $149.0^{\circ} \mathrm{E}$ & -0.0074 & -0.0107 & $-0.0008^{\star}$ & -0.0089 \\
\hline Christchurch & $43.6^{\circ} \mathrm{S}$ & $172.8^{\circ} \mathrm{E}$ & -0.0073 & -0.0108 & +0.0015 & -0.0071 \\
\hline
\end{tabular}
ues are significantly different from zero at a $95 \%$ confi-

Table 1. Geographic coordinates of the Southern Hemisphere ionospheric stations here analyzed and linear foF 2 trend in MHz/year calculated for four different conditions: filtering seasonal and solar activity effects using Rz (4th and 6th columns) and F10.7 (5th and 7th columns), and for periods covering solar cycles 18 to 21 (4th and 5th columns) and 18 to 23 (6th and 7th columns) (values with * mean not significantly different from 0 ). 
dence level. The significance was assessed calculating the statistic $\mathrm{t}$ as $\alpha / \sigma$, where

$$
\mathrm{t}=\frac{\alpha}{\sigma}=\frac{\alpha}{\frac{\alpha}{\sqrt{\mathrm{n}-2}} \sqrt{\frac{1}{\mathrm{r}^{2}}-1}}=\mathrm{r} \frac{\sqrt{\mathrm{n}-2}}{\sqrt{1-\mathrm{r}^{2}}}
$$

$\sigma$ is the standard deviation of trend and $\mathrm{r}$ the correlation coefficient of the regression between foF $2_{\text {res }}$ and time.

$\mathrm{Rz}$ was chosen as solar EUV proxy instead of F10.7, which is the one recommended [Lastovicka et al. 2012, Elias 2014], since F10.7 is not available since the beginning of cycle 18. In addition, since we are not including here cycle 23, the difference between filtering solar activity using Rz or F10.7 should not be large. As a test, we linearly extrapolated F10.7 values to 1944, using a linear regression between $\mathrm{Rz}$ and F10.7 for the period 1947-1986. Trends, listed in Table 1, were assessed following the same steps as in $\mathrm{Rz}$ case. It can be noticed that although the difference is $\sim 40-50 \%$ for the three stations, they are all significant decreasing trends.

\subsection{Extending period to cycle 23}

The three stations here analyzed have available data records until year 2009. However two of them, Christchurch and Brisbane, present a big data gap during solar cycle 22 , which cannot be easily interpolated for trend detection purposes. As a first approach, without interpolation, trends for the period covering cycles 18 to 23 were estimated.

Special care should be taken in foF 2 filtering process when solar cycle 23 is included [Elias et al. 2014]. The period 2007-2009 that corresponds to minimum activity level, is characterized by lower EUV solar radiation than previous solar cycles [Solomon et al. 2013]. In addition it is different than that deduced from traditional solar EUV proxies as Rz and F10.7. Emmert et al. [2010] suggested that the long-term relationship between EUV irradiance and F10.7 has changed markedly since around 2006 with EUV levels decreasing more than expected from F10.7 proxy. Rz instead, underestimates EUV during the maximum and declining phase of the cycle [Lukianova and Mursula 2011]. In addition, Rz and F10.7, which were used interchangeably as EUV proxy, present a significant change in their relationship since solar cycle 23 [Tapping and Valdes 2011].

Taking all this into account, trends were estimated for each station considering $\mathrm{Rz}$ and F0.7 to filter solar activity effect. Trend values are listed in Table 1. Figure 2 shows foF $2_{\text {res }}$ together with the fitted linear trend, considering both $\mathrm{Rz}$ and F10.7 separately as solar activity filters. It can be clearly noticed that the inclusion of solar cycle 23 marks a significant differ-
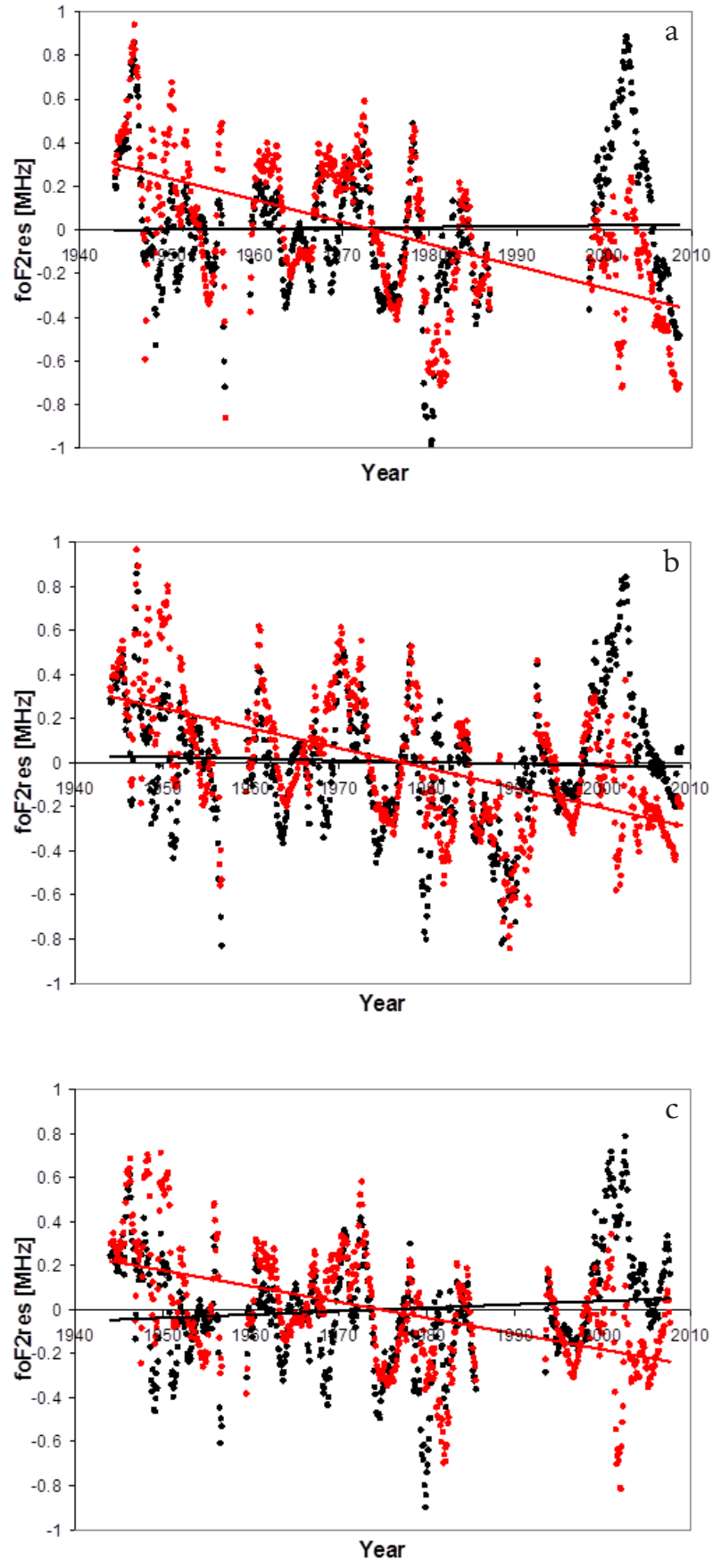

Figure 2. foF2 residual for (a) Brisbaen $\left(27.5^{\circ} \mathrm{S}, 152.9^{\circ} \mathrm{E}\right)$, (b) Canberra $\left(35.3^{\circ} \mathrm{S}, 149.0^{\circ} \mathrm{E}\right)$ and (c) Christchurch $\left(43.6^{\circ} \mathrm{S}, 172.8^{\circ} \mathrm{E}\right)$, after filtering seasonal and solar activity effects using $\mathrm{Rz}$ (black dots) and F10.7 (red dots) as solar EUV proxy and fitted linear trend (corresponding solid lines).

ence not only trend values, but also in the sign, between both EUV proxies.

\section{Geomagnetic activity effect}

Danilov and Mikhailov [1999], Mikhailov and Marin [2000] and Danilov [2002] with a new approach, obtained a dependence of the trend magnitude on geomagnetic latitude (with more negative values at higher latitudes) indicating that F2-layer trends might be related to long-term changes in geomagnetic activity and 
F2-layer storm mechanisms. They show those periods with negative and positive foF 2 trends exist, which correspond to the periods of long-term increasing or decreasing geomagnetic activity. However, more recently, according to Danilov [2012], the assumptions made at the end of 1990's that the observed trends in ionospheric parameters are a manifestation of secular changes in geomagnetic activity could be proved only for the data before 1980-1990.

In our case, the three stations here analyzed are mid-latitude stations, so the geomagnetic activity effect should not be large. To prove this, aa index was added to Equation (1) to filter foF2, that is

$$
\mathrm{foF} 2_{\text {res }}=\mathrm{foF} 2_{\exp }-(\mathrm{a}+\mathrm{b} \mathrm{Rz}+\mathrm{c} \text { aa })
$$

This index is a global geomagnetic activity index which measures the disturbance level of the Earth's magnetic field. The index, in nT units, is available from http:/ / www.wdcb.ru/stp/data/geomagni.ind/aa/ and from http://isgi.cetp.ipsl.fr/source/indices/.

Following the same steps described in Section 2.2, trends were assessed considering period 1944-1986, and 1944-2009, using Rz and F10.7 as solar EUV proxies separately. Trend values are shown in Table 2 . As can be noticed, in all the cases trend values are almost the same as those seen in Table 1. This can be expected since the correlation coefficient between foF 2 and $\mathrm{Rz}$ or F10.7, is already greater than 0.97 meaning that $\mathrm{Rz}$ (or F10.7) explain more than $94 \%$ of foF 2 variance in all the cases. Including aa into the linear regression model, only increases the correlation by $\sim 0.005$, that is it adds only $1 \%$ to the explained variance.

Taking into account the result of Danilov [2012] that there is a different foF2 negative trend after $\sim 1980$ 1990, and of Lastovicka et al. [2012] that observes a change of dependency of F2 trends after 2000, we made a rough analysis in the case of Canberra, which presents complete data set covering the period 19442009. We estimated the aa coefficient, $c$ in Equation (4), considering F10.7 as EUV proxy, for the period 1944-
1989 and 1990-2009 separately. In the first case it results $-0.15 \pm 0.03$ and in the latter $-0.04 \pm 0.03$, while $b$ results $0.395 \pm 0.003$ and $0.382 \pm 0.003$. That is, after 1990 the coefficient of aa is almost null. This could be evidence of a decline in importance of aa, however when we look at trends estimated with and without aa in foF2 filtering, we cannot arrive to a definitive conclusion through this type of analysis. Anyway as it has been observed by Bremer [1992, 1998] and Mikhailov and Marin [2000] the inclusion of a geomagnetic index in the regression in fact does not remove the geomagnetic dependence.

\section{Earth's magnetic field effect}

The possibility of ionospheric trends induced by the Earth's magnetic field secular variations was first suggested by Foppiano et al. [1999], and followed then by other papers [Elias 2009, Cnossen 2014]. Earth's magnetic field, generated in the Earth's core, presents long term variations in the field's strength and orientation. A simple mechanism through which trends in the Earth's magnetic field would affect the ionosphere is through changes in the dip angle (I). The $\sin (\mathrm{I}) \cos (\mathrm{I})$ factor, associated to effects of neutral winds on hmF2 [Rishbeth 1998] will also change. The horizontal thermospheric wind $U$ drives ions and electrons, up during the night and down during the day, along the geomagnetic field lines at speed $U \cos (\mathrm{I})$. The vertical component $\mathrm{U} \sin (\mathrm{I}) \cos (\mathrm{I})$ raises the F2-peak during night time (when U blows from Pole to Equator) and lowers it during daytime (when $U$ blows from Equator to Pole), increasing or decreasing the peak electron density. An increase in the $\sin (\mathrm{I}) \cos (\mathrm{I})$ factor would produce an additional lowering of the F-region with a decrease in foF2, during daytime, and an additional raise of the region with an increase in foF 2 during the night. A decrease in the $\sin (\mathrm{I}) \cos (\mathrm{I})$ factor would produce the opposite effect.

The region of strongest variations of foF 2 , lies between $10^{\circ} \mathrm{N}$ and $30^{\circ} \mathrm{S}$ in latitude and between $20^{\circ} \mathrm{E}$ and $80^{\circ} \mathrm{W}$ in longitude, which is also the region of strongest changes in I and $\sin (\mathrm{I}) \cos (\mathrm{I})$ factor [Elias 2009, Cnossen

foF2 trend $[\mathrm{MHz} /$ year $]$

\begin{tabular}{lcccc}
\cline { 2 - 4 } Station & \multicolumn{2}{c}{ Period cycles 18-21 } & \multicolumn{2}{c}{ Period cycles 18-23 } \\
\cline { 2 - 4 } \cline { 3 - 4 } Brisbaen & $\mathrm{Rz}+\mathrm{aa}$ & $\mathrm{F} 10.7+\mathrm{aa}$ & $\mathrm{Rz}+\mathrm{aa}$ \\
Canberra & -0.0083 & -0.0118 & $+0.0001^{\star}$ \\
Christchurch & -0.0068 & -0.0105 & $-0.0007^{\star}$ & -0.0089 \\
\hline
\end{tabular}

Table 2. foF2 trend in $\mathrm{MHz}$ / year calculated for four different conditions: filtering seasonal and solar activity effects using Rz and aa (4th and 6th columns) and F10.7 and aa ( 5 th and 7th columns), and for periods covering solar cycles 18 to 21 (4th and 5 th columns) and 18 to 23 ( 6 th and 7 th columns) (values with * mean not significantly different from 0 ). 
2014]. At the mid-latitude zone, corresponding to the locations of the ionospheric stations here analyzed, the expected trends due to changes in I, should not be statistically significant different from zero.

A qualitative analysis can be made through the $\sin (\mathrm{I}) \cos (\mathrm{I})$ factor. Figure 3 presents the time variation of this factor calculated from the International Geomagnetic Reference Field (IGRF) model (http:/ / ccmc. gsfc.nasa.gov/modelweb/models/igrf_vitmo.php), and for the three stations here analyzed, it can be noticed an increase for the period 1944-1986.

This implies that although it is very small, an increasing trend in daytime foF 2 , is contrary to the values obtained from measured foF 2 data.

\section{Conclusions}

All stations present a decreasing trend in foF 2 during the period 1944-1986, covering complete solar cycles from cycle 18 to 21 , consistent with the consequences expected in the ionosphere $\mathrm{F} 2$ region due to the increased concentration of greenhouse gases.

For a doubling of $\mathrm{CO}_{2}$ concentration Qian et al. [2009] obtain a maximum decrease of $18 \%$ to $20 \%$ in foF2. Making a linear extrapolation to the actual increase in $\mathrm{CO}_{2}$ during the period analyzed in this paper, and assuming an average foF 2 value of $10 \mathrm{MHz}$, a foF2 decrease of $-0.005 \mathrm{MHz} /$ year should be expected. According to the values here obtained when $\mathrm{Rz}$ is used as solar EUV proxy ( $\sim 0.008 \mathrm{MHz} /$ year), the greenhouse effect would account for $60 \%$ of these trends. When F10.7 is used as EUV proxy, extrapolating to cover the complete period 1944-1986, this account diminish to $50 \%$.

With regard to natural forcings, in the case of longterm variation in geomagnetic activity, our results hardly vary when considering the aa index as another linear term in Equation (1). Probably in a different kind of analysis, and considering the result of Danilov [2012] this could be more noticeable. In the case of secular variations of the intrinsic magnetic field of the Earth, its ability to induce trends depends on the geographic location. The values of the expected trends are very small (even lower than those expected by anthropogenic effect) except in the region between $10^{\circ} \mathrm{N}$ and $40^{\circ} \mathrm{S}$ and $60^{\circ} \mathrm{W}$ and $10^{\circ} \mathrm{E}$ [Elias 2009, Cnossen 2014]. The stations considered are located outside this region. In addition a rough qualitative estimation of the expected effect does not even agree in the sign of the expected trends.

When using the complete data sets that go until solar cycle 23 , the most reliable trends are those obtained using F10.7 as EUV proxy [Lastovicka et al. 2012, Elias 2014]. Trend values are close to those corresponding to period 1944-1986, but less negative, very likely due to a lack of agreement between F10.7 and the solar

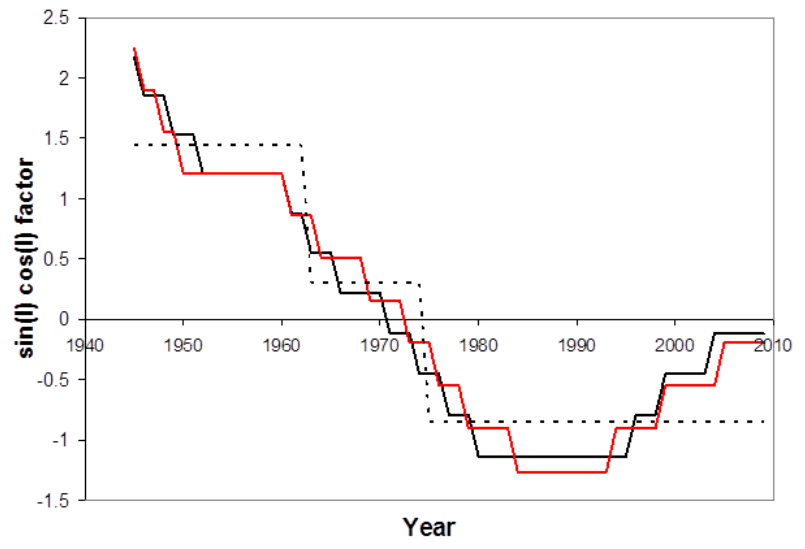

Figure 3. Standardized $\sin (\mathrm{I}) \cos (\mathrm{I})$ factor estimated with International Geomagnetic Reference Field (IGRF) model (http: / / ccmc.gsfc.nasa. gov/modelweb/models/igrf_vitmo.php) for Brisbaen (black solid line), Canberra (red solid line) and Christchurch (dashed black line).

EUV radiation which F10.7 is supposed to represent.

The importance of understanding and measuring trends in the upper atmosphere lies in:

1) the importance of understanding and predicting the behavior of the whole atmosphere, which is essential to human life.

2) the effect of the decreasing density on space debris. There are approximately 22,500 objects larger than $10 \mathrm{~cm}$ in Earth orbit, and $95 \%$ of them is classified as "space debris". These objects represent a significant risk to spacecraft operations due to possible collisions. Recent studies [Lewis et al. 2011] suggest that the decrease in the density of the upper atmosphere makes the space debris remains in orbit longer than expected.

The results obtained here are intended to be a contribution to both topics.

\section{References}

Bremer, J. (1992). Ionospheric trends in mid-latitudes as a possible indicator of the atmospheric greenhouse effect, J. Atmos. Terr. Phys., 54, 1505-1511.

Bremer, J. (1998). Trends in the ionospheric E and F regions over Europe, Ann. Geophysicae, 16, 986-996.

Chen, Y., L. Liu and W. Wan (2011). Does the F10.7 index correctly describe solar EUV flux during the deep solar minimum of 2007-2009?, J. Geophys. Res., 116; doi:10.1029/2010JA016301.

Cnossen, I. (2014). The importance of geomagnetic field changes versus rising $\mathrm{CO}_{2}$ levels for long-term change in the upper atmosphere, J. Space Weather Space Clim., 4, A18.

Damboldt, T., and P. Suessmann (2012). Consolidated Database of Worldwide Measured Monthly Medians of Ionospheric Characteristics foF2 and M(3000)F2, INAG Bulletin on the Web, INAG-73; www.ips.gov. au/IPSHosted/INAG/web-73/2012/damboldt_con solidated_database.pdf. 
Danilov, A.D., and A.V. Mikhailov (1999). Long-term trends in the parameters of the F2- region: A new approach, Geom. Aeron., 39, 473-479.

Danilov, AD. (2002). Overview of the trends in the ionospheric E and F2 regions, Phys. Chem. Earth, 27, 579-588.

Danilov, A.D. (2012). Long-Term Trends in the Upper Atmosphere and Ionosphere (a Review), Geom. Aeron., 52, 271-291.

Elias, A.G. (2009). Trends in the F2 ionospheric layer due to long-term variations in the Earth's magnetic field, J. Atmos. Solar Terr. Phys., 71, 1602-1609.

Elias, A.G. (2014). Filtering ionosphere parameters to detect trends linked to anthropogenic effects, Earth, Planets and Space, 66, 113.

Elias, A.G., B.F. de Haro Barbas, S. Kiyoto and J.R Souza (2014). Effect of solar cycle 23 in foF2 trend estimation, Earth, Planets and Space, 66, 111.

Emmert, J.T., J.L. Lean and J.M. Picone (2010). Recordlow thermospheric density during the 2008 solar minimum, Geophys. Res. Lett., 37; doi:10.1029/2010 GL043671.

Foppiano, A.J., L. Cid and V. Jara (1999). Ionospheric long-term trends for South American mid-latitudes, J. Atmos. Solar Terr. Phys., 61, 717-723.

Lastovicka, J., S.C. Solomon and L. Qian (2012). Trends in the Neutral and Ionized Upper Atmosphere, Space Sci. Rev., 168, 113-145.

Lewis, H.G., A. Saunders, G. Swinerd and R.J. Newland (2011). Effect of thermospheric contraction on remediation of the near Earth space debris environment, J. Geophys. Res., 116.

Lukianova, R., and K. Murusla (2011). Changed relation between sunspot numbers, solar UV / EUV radiation and TSI during the declining phase of solar cycle 23, J. Atmos. Solar Terr. Phys., 73, 235-240.

Mikhailov, A.V., and D. Marin (2000). Geomagnetic control of the foF2 long-term trends, Ann. Geophysicae, 18, 653-665.

Qian, L., A.G. Burns, S.C. Solomon and R.G. Roble (2009). The effect of carbon dioxide cooling on trends in the F2-layer ionosphere, J. Atmos. Solar Terr. Phys., 71, 1592-1601.

Rishbeth, H. (1990). A greenhouse effect in the ionosphere?, Planet. Space Sci., 38, 945-948.

Rishbeth H. (1998). How the thermospheric circulation affects the ionospheric F2 layer, J. Atmos. Solar Terr. Phys., 60, 1385-1402.

Roble, R.G., and R.E. Dickinson (1989). How will changes in carbon dioxide and methane modify the mean structure of the mesosphere and thermosphere?, Geophys. Res. Lett., 16, 1441-1444.

Solomon, S.C., L. Qian and A.G. Burns (2013). The anomalous ionosphere between solar cycles 23 and 24, J. Geophys. Res., 118, 6524-6535.

Tapping, K.F., and J.J. Valdes (2011). Did the Sun change its behavior during the decline of cycle 23 and into cycle 24?, Sol. Phys., 272, 337-350.
Corresponding author: Blas F. de Haro, Universidad Nacional de Tucumán, Departamento de Física, Tucumán, Argentina; email: blasdeharo2000@yahoo.com.ar.

(C) 2015 by the Istituto Nazionale di Geofisica e Vulcanologia. All rights reserved. 